\title{
Acid suppression and gastric atrophy: sifting fact from fiction
}

\author{
R M Genta
}

\begin{abstract}
Summary
Prolonged pharmacological acid suppression is associated with various histological changes in the gastric mucosa, particularly in Helicobacter pylori infected patients. In a number of subjects these changes include a shift in the gastric inflammation from the antrum to the corpus. This finding has been interpreted as gastric atrophy, and the possibility that acid suppression accelerates the progress of lesions that may lead to gastric cancer has been considered. Two recent studies on the relation between treatment with proton pump inhibitors and atrophic gastritis have yielded apparently contradictory results. These studies are reviewed in detail here and some of the possible reasons for the discrepant conclusions are explored. In particular, the way the terms "gastric atrophy" and "atrophic gastritis" are used is examined critically.
\end{abstract}

\section{What is gastric atrophy?}

As seen in histological preparations, the mucosa of the normal gastric corpus consists of a layer of mucous cells invaginating into shallow pits, which merge with the oxyntic glands. The latter are separated by little or no extracellular matrix in which few mononuclear cells may be interspersed (fig 1A). When the mucosa is infiltrated by inflammatory cells (mostly plasma cells and lymphocytes, in the case of $H$ pylori infection) the glands become separated and pushed aside, and may become invisible, or "lost" (fig 1B). In some circumstances glands may be destroyed and substi-

R M Genta

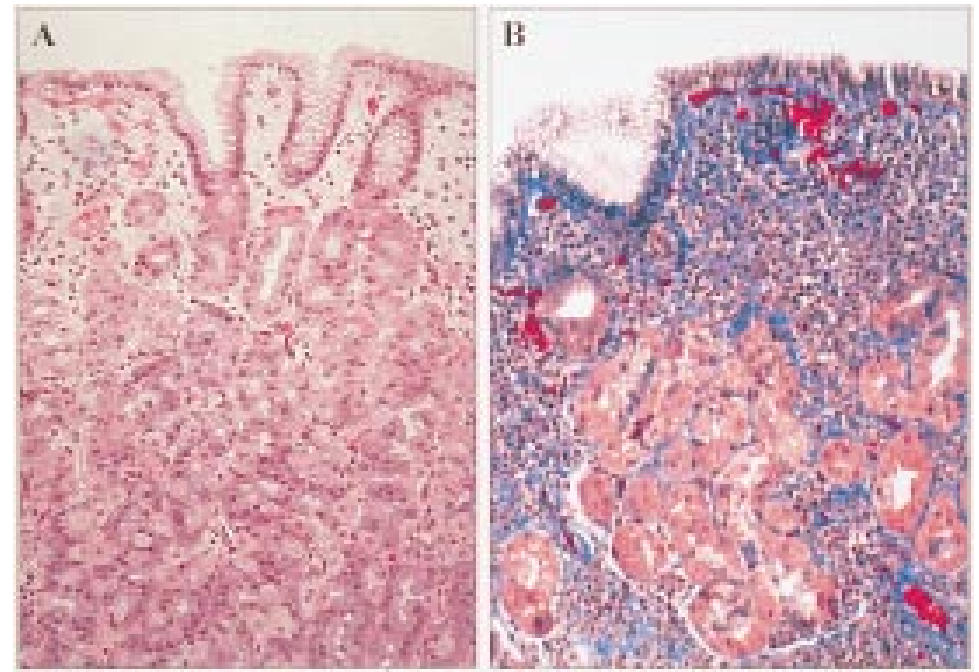

Figure 1 (A) Normal gastric corpus mucosa. Glands are tightly packed and there are few inflammatory cells in the subepithelial area and in between the glands. (B) Severe corpus gastritis with probable atrophy. Islands of glands are separated by large numbers of inflammatory cells and fibrous tissue. tuted with either intestinal metaplastic epithelium or fibroblasts and extracellular matrix, or a combination of the two. In such cases the glands are invisible for the obvious reason that they are not there- - that is, they are truly "lost". Most definitions of gastric atrophy, including Whitehead's, ${ }^{1}$ Correa's, ${ }^{2}$ the original Sydney System, ${ }^{3}$ and the updated Sydney System ${ }^{4}$ have emphasised gland loss without specifying what replaces the missing glands. Therefore, mucosa showing the histopathological features depicted in figure $1 \mathrm{~B}$ would be considered atrophic by many observers, but not by those who would want to know whether the missing glands are really lost or just displaced by the inflammation. Functional studies do not help clarify the issue: inflammation in the oxyntic mucosa interferes with the somatostatingastrin axis and depresses acid production. ${ }^{56}$ Of course, the destruction of oxyntic glands results in hypochlorhydria. Consequently, these two types of gland loss (one apparent and one real, but both with similar pathophysiological consequences) have not been considered as separate entities in studies that have looked at the relation between structure and function of the gastric mucosa. In the absence of stringent histopathological criteria, it is hardly surprising that pathologists have generally failed to agree with one another in the evaluation of atrophy. ${ }^{7}$

\section{The importance of being atrophic}

Soon after $H$ pylori was recognised as the cause of chronic gastritis, it became apparent that the epidemiological paradigms that linked atrophic gastritis and gastric cancer could be applied to $H$ pylori infection. ${ }^{8-10}$ Populations with a high prevalence of $H$ pylori infection and atrophic gastritis (such as Colombia ${ }^{11}$ and Japan ${ }^{12}$ ) have a high incidence of gastric cancer; in contrast, populations with low prevalence of $H$ pylor $i^{13}$ or low prevalence of atrophic gastritis ${ }^{14}{ }^{15}$ have low a incidence of gastric cancer. Furthermore, several studies have shown quite convincingly that long-standing $H$ pylori infection results in atrophy and intestinal metaplasia in a portion of the infected subjects. ${ }^{16}$

The association between atrophic gastritis and gastric cancer detected in pre-helicobacter studies could just as well be applied to $H$ pylori infection: no longer gastritis, but its principal cause- $H$ pylori - could be named as the carcinogen, and both epidemiological and biological evidence became available to support this role.

The former consist of a large body of data showing that infection with $H$ pylori increases the cancer risk between three- and 10-fold, depending on the population studied. ${ }^{17}{ }^{18}$ The 
latter include a broad variety of observations linking particular histopathological features of the gastric mucosa with the development of intestinal-type adenocarcinoma. ${ }^{9}{ }^{19-21}$ These histopathological features include alterations of the mucosal architecture, loss of glands, fibrosis, and replacement of epithelial elements by an intestinal-type metaplastic epithelium ("intestinal metaplasia"). The constellation of these features has been variously called "atrophic gastritis" and "gastric atrophy," but these terms remain poorly defined and, consequently, loosely used. ${ }^{22}$ The same lack of agreement that exists on the definition of atrophy also exists about the concept of atrophic gastritis. What is atrophic gastritis? What is the atrophic gastritis considered a precursor of gastric cancer? The classic studies that defined atrophic gastritis (also called "multifocal atrophic gastritis") were performed on gastrectomy specimens. With the entire stomach available for examination it was possible to see large patches of atrophic and metaplastic epithelium extending proximally and distally along the lesser curvature. The extension of these patches was so obvious that there appeared to be no need for a strict topographical definition. In the past two decades, however, effective antacid therapies and treatment for $H$ pylori have greatly reduced the need for gastrectomy and, consequently, the availability of resected stomachs for histopathological examination. The diagnosis of atrophic gastritis must now be made based on the examination of a limited number of biopsy specimens that, even in the most audacious sampling protocols, ${ }^{23}$ still represent only a minimal portion of the gastric mucosa. When only two or three samples from the stomach are available for examination it may be imprudent to make a diagnostic statement which has much broader topographical implications, such as "multifocal atrophic gastritis." Although it is very difficult to produce an accurate topographical definition of atrophic gastritis (the multifocal type associated with chronic $H$ pylori infection, unknown environmental factors, and gastric cancer), the best approximation can only result from studies involving the observation of multiple mapped biopsy samples. If such a definition is generated, tested, and found to be satisfactory, it should then be disseminated and pathologists could be encouraged to use it.

\section{The Dutch Study}

Based on the observation that profound suppression of gastric acid is associated in some subjects with increased severity of gastritis caused by $H$ pylori, the authors hypothesised that acid suppression might also increase the risk of atrophic gastritis.

To test their hypothesis, the authors studied patients from two separate cohorts who were being treated for reflux oesophagitis. ${ }^{24}$ One cohort consisted of 72 Swedish patients (mean age 53 years) treated with fundoplication who did not receive acid suppressive therapy after their operation. The second cohort consisted of 105 Dutch patients (mean age 62 years) treated with omeprazole (20 to $40 \mathrm{mg}$ once daily). The presence of $H$ pylori was assessed at the first visit by histological evaluation in the fundoplication group and by histological and serological evaluation in the omeprazole group; however, none of the patients was treated for $H$ pylori infection. In both cohorts, the patients were followed for three to eight years; all patients underwent a gastroscopy at the beginning of the study as well as repeated gastroscopies during the follow up period. At each gastroscopy biopsy specimens were obtained from the gastric corpus for blinded histological evaluation. The histological examination included the semi-quantitative evaluation of the features of $H$ pylori gastritis according to the Sydney System. ${ }^{3}$ The criteria used by the authors for the diagnosis of atrophic gastritis were not stated. However, they did state that among the patients treated with fundoplication, atrophic gastritis did not develop in any of the 31 who were infected with $H$ pylori at baseline or the 41 who were not infected. Among the patients treated with omeprazole, none of whom had atrophic gastritis at baseline, atrophic gastritis developed in 18 of the 59 infected with $H$ pylori and two of the 46 who were not infected. None of the patients in either group developed intestinal metaplasia. Based on these observations, the authors concluded that patients with reflux oesophagitis and $H$ pylori infection who are treated with omeprazole are at increased risk of atrophic gastritis.

\section{The Swedish study}

In this study, patients with chronic gastrooesophageal reflux were initially treated with $20-40 \mathrm{mg} /$ day omeprazole for $<4$ months to control symptoms and heal the oesophagitis. ${ }^{25}$ Patients who had symptom relief were then randomised and allocated to either omeprazole maintenance therapy or antireflux surgery without vagotomy. All patients underwent gastroscopy at the beginning of the study as well as during the follow up period, which lasted approximately 3.5 years. At each endoscopy, multiple mucosal biopsy specimens were obtained from the gastric corpus according to a pre-determined protocol. The biopsy specimens were then examined blindly by a single pathologist, who scored the intensity of the $H$ pylori infection and the extension of intestinal metaplasia according to the criteria established by the Sydney System, ${ }^{3}$ but the degree of glandular atrophy according to Whitehead et al. ${ }^{26}$

In this study 155 patients were randomised to the omeprazole treatment: 116 were men and 39 women; their median age was 54 years; 66 of these patients had $H$ pylori infection at the initial examination; 151 patients were randomised to the antireflux surgery: 116 were men and 35 women; their median age was 51 years; 73 of these patients had $H$ pylori infection.

None of the patients without $H$ pylori infection in either randomisation arm developed atrophy during the follow up period. In the group on omeprazole, of the $62 \mathrm{H}$ pylori infected patients who had no atrophy at the 


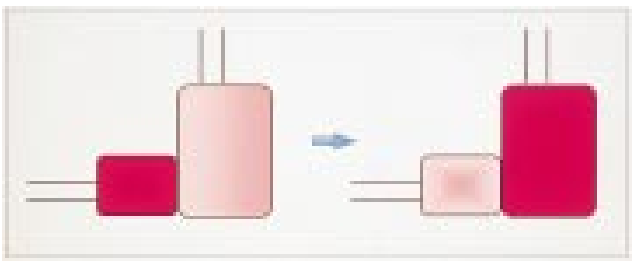

Figure 2 In $H$ pylori infected patients who receive proton pump inhibitors for extended periods the pattern of gastritis changes from antral predominant to corpus predominant. The bacterial density tends to decrease in the antrum whereas it remains essentially unchanged in the corpus.

beginning of the study, five developed mild/ moderate atrophy and three developed severe atrophy. In this group, two patients had mild/moderate atrophy at the beginning of the study; at the end of the study, one of them had no atrophy, whereas the other one had severe atrophy. Two subjects who had mild/moderate atrophy remained unchanged, and of the two with severe atrophy initially, one remained unchanged whereas the other became normal. Similarly to the Dutch study, no patient in either group developed any intestinal metaplasia.

The authors concluded that long term omeprazole therapy was no different from fundoplication in its effects on the development of gastric atrophy. Furthermore, they stated that, as no intestinal metaplasia developed in any of these patients and only atrophic gastritis associated with intestinal metaplasia is considered a precursor of gastric cancer, there is no evidence to support the hypothesis that treatment with proton pump inhibitors in subjects with $H$ pylori infection may increase the risk of gastric cancer.

\section{Reactions}

The authors of the Dutch study had previously investigated the same issue and had found that long term proton pump inhibitors in patients with $H$ pylori infection significantly decreased the inflammation and bacterial colonisation in the antrum, leading to negative antral cultures in $61 \%$ (20 of 33) patients. In contrast, the inflammation of the corpus mucosa significantly increased despite stable bacterial counts. ${ }^{27}$ The phenomenon, depicted schematically in figure 2 , is commonly observed in clinical practice. Although its significance

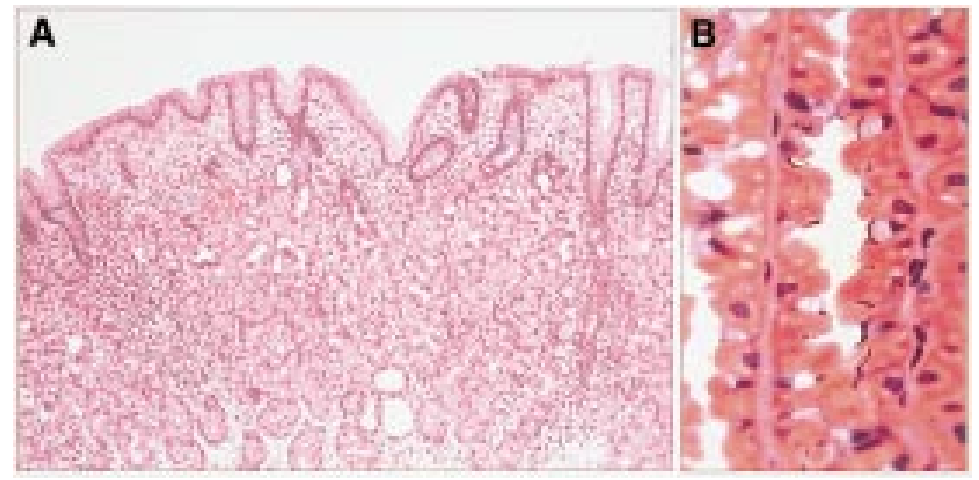

Figure 3 (A) Moderate corpus gastritis, oxyntic gland dilatation and "pseudo-hyperplasia" in a $H$ pylori infected patient receiving proton pump inhibitors. (B) At high magnification, $H$ pylori can be seen in the deep portion of the oxyntic glands and within the canaliculi of the parietal cells. remains unclear, the authors' suggestion that $H$ pylori infected patients in need of long term acid suppressive therapy should receive bacterial eradication therapy seems sensible and has been espoused by a recent European Consensus Conference held in Maastricht. ${ }^{28}$ However, the unexplained leap from the term "corpus gastritis" (used in the first article) to "atrophic gastritis" (used, but not defined, in the second paper $^{24}$ and an editorial that followed a few months later ${ }^{29}$ caused a great degree of consternation among regulatory agencies in the United States. The question of whether proton pump inhibitors may accelerate the progression of preneoplastic lesions in the stomach of $H$ pylori infected subjects was submitted to an advisory panel. The panel concluded that the evidence was insufficient and that no suggestions for change in the usage of proton pump inhibitor therapy should be made.

\section{What proton pump inhibitors really do to the gastric mucosa}

In addition to the shift from a predominately antral to a predominantly corpus inflammation that occurs roughly in half of the $H$ pylori infected patients on long term proton pump inhibitors (fig $3 \mathrm{~A}),{ }^{27}{ }^{30-33}$ in a number of subjects on long term proton pump inhibitors the oxyntic mucosa (irrespective of the $H$ pylori status) acquires a characteristic appearance, variously described as "hypertrophic," "pseudo-hypertrophic," or "hyperplastic" (fig $3 \mathrm{~A}) .^{34-36}$ Recently, however, this change has been described also in patients with gastric ulcer apparently not receiving acid suppressing therapy. ${ }^{37}$ In a small percentage of $H$ pylori infected individuals (perhaps less than 10\%), bacteria become visible in the deeper third of the oxyntic glands, and sometimes they appear to be lodged inside the canaliculi of the parietal cells (fig 3B). No increase in the deep mucosal inflammatory response is seen in these cases. ${ }^{38}$ The significance of this curious finding is unclear.

Another effect of prolonged treatment with proton pump inhibitors which is believed to occur in less than $10 \%$ of patients without $H$ pylori infection and in up to $30 \%$ of those with the infection is the development of significant argyrophil cell hyperplasia. ${ }^{39}{ }^{40}$ This is not, however, accompanied by an increased risk of developing malignant carcinoids.

\section{Conclusions}

Although the issues discussed in this article are apparently disparate, they all revolve around one poorly understood concept, the concept of atrophy. If a clear definition existed and a set of criteria were established for the diagnosis of atrophic gastritis, many of the confusing and sometimes acrimonious debates that originated from the study of the $H$ pylori infected mucosa in subjects treated with proton pump inhibitors would have been avoided.

With this aim in mind, a group of pathologists, gastroenterologists, and gastrointestinal physiologists with a commitment to the study of gastric atrophy gathered in Houston, Texas, in February 1998. Their aim was to agree on 
one usable definition of atrophy and one of atrophic gastritis and to disseminate these definitions to a wide audience of gastroenterologists and pathologists. The deliberations and conclusions of the meeting will be reported in a major pathology journal.

1 Whitehead R. Gastritis-histopathological background. Scand $\mathcal{F}$ Gastroenterol 1982;79(suppl):40-3.

2 Correa P. Chronic gastritis: a clinico-pathological classification. Am $\mathcal{F}$ Gastroenterol 1988;83:504-9.

3 Price AB. The Sydney System: histological division. 7 Gas troenterol Hepatol 1991;6:209- 22

4 Dixon MF, Genta RM, Yardley JH, et al. Classification and grading of gastritis. The updated Sydney System. International Workshop on the Histopathology of Gastritis, Houston 1994. Am F Surg Pathol 1996;20:1161-81.

5 Calam J. Helicobacter pylori, acid and gastrin. Eur $\mathcal{F}$ Gastroenterol Hepatol 1995;7:310-17.

6 Calam J. The somatostatin-gastrin link of Helicobacter pylori infection. Ann Med 1995;27:569-73.

pylori infection. Ann Med 1995;27:569-73.
7 El-Zimaity HM, Graham DY, al-Assi MT, et al. InterobEl-Zimaity HM, Graham DY, al-Assi MT, et al. Interobserver variation in the histopathological asse

8 Siurala M, Lehtola J, Ihamäki T. Atrophic gastritis and its sequelae. Results of 19-23 years' follow-up examinations. Scand $\mathcal{F}$ Gastroenterol 1974;9:441-6.

9 Correa P. Chronic gastritis as a cancer precursor. Scand $\mathcal{F}$ Gastroenterol 1984;104(suppl):131-6.

10 Sipponen P, Kekki M, Haapakoski J, et al. Gastric cancer risk in chronic atrophic gastritis: statistical calculations of cross-sectional data. Int $\mathcal{F}$ Cancer 1985;35:173-7.

11 Correa P, Haenszel W, Cuello C, et al. Gastric precancerous process in a high risk population: cross-sectional studies. Cancer Res 1990;50:4731-6.

12 Asaka $M$, Kato $M$, Kudo $M$, et al. Relationship between Helicobacter pylori infection, atrophic gastritis and gastric
carcinoma in a Japanese population. Eur $\mathcal{f}$ Gastroenterol Hepatol 1995;7(suppl 1):S7-10.

13 Andersen LP, Gaarslev K. IgG subclass antibodies against Helicobacter pylori heat-stable antigens in normal persons Helicobacter pylori heat-stable antigens in normal pers
and in dyspeptic patients. APMIS 1992;100:747-51.

14 Holcombe C, Omotara BA, Eldridge J, et al. H. pylori, the most common bacterial infection in Africa: a random seromost common bacterial infection in Africa: a ran

15 Holcombe C, Umar H, Lucas SB, et al. Low incidence of clinically significant gastroduodenal pathology despite a high incidence of Helicobacter pylori infection. Trans $R$ So Trop Med Hyg 1994;88:569-71.

16 Kuipers EJ, Uyterlinde AM, Pena AS, et al. Long-term sequelae of Helicobacter pylori gastritis. Lancet 1995;345 1525-8.

17 Forman D. Helicobacter pylori and gastric cancer. Scand $\mathcal{f}$ Gastroenterol 1996;215(suppl 1):48-51.

18 Parsonnet J, Friedman GD, Vandersteen DP, et al. Helicobacter pylori infection and the risk of gastric carcinoma. N Engl f Med 1991;325:1127-31.

19 Correa P. A human model of gastric carcinogenesis. Cancer Res 1988;48:3554-60.

20 Nomura A, Yamakawa $\mathrm{H}$, Ishidate $\mathrm{T}$, et al. Intestinal metaplasia in Japan: association with diet. 7 Natl Cancer Inst plasia in Japan:
1 Stemmermann GN. Intestinal metaplasia of the stomach. A status report. Cancer 1994; 74:556-64.

22 Genta RM. Recognizing atrophy: another step toward a classification of gastritis. Am F Surg Pathol 1996;20 (suppl 1):S23-30.

23 Genta RM, Graham DY. Comparison of biopsy sites for the histopathologic diagnosis of Helicobacter pylori: a topographic study of $\mathrm{H}$. pylori density and distribution. Gastrointest Endosc 1994;40:342-5.

24 Kuipers EJ, Lundell L, Klinkenberg-Knol EC, et al. Atrophic gastritis and Helicobacter pylori infection in ptrophic gastritis and Helicobacter pylori infection in fundoplication. N Engl ₹ Med 1996;334:1018-22.

25 Lundell L, Havu N, Anderson A, et al. Gastritis development and acid suppression therapy revisited: results of a randomized clinical study with long-term follow-up [abstract]. Gastroenterology 1997;112:A28.

26 Whitehead R, Truelove SC, Gear MW. The histological diagnosis of chronic gastritis in fibreoptic gastroscope biopsy specimens. F Clin Pathol 1972;25:1-11.

27 Kuipers EJ, Uyterlinde AM, Pena AS, et al. Increase of Helicobacter pylori-associated corpus gastritis during acid suppressive therapy: implications for long-term safety. $A m \mathcal{F}$ pressive therapy: implications

28 Malfertheiner P, Megraud F, O'Morain C, et al. Current European concepts in the management of Helicobacter pylori infectionw-the Maastricht Consensus Report. Eur f Gastroenterol Hepatol 1997;9:1-2.

29 Parsonnet J. Helicobacter pylori in the stomach — a paradox unmasked. N Engl f Med 1996;335:278-80.

30 Dickey W, Kenny BD, McConnell JB. Effect of proton pump inhibitors on the detection of Helicobacter pylori in gastric biopsies. Aliment Pharmacol Ther 1996;10:289-93.

31 Uemura N, Oomoto Y, Mukai T, et al. Gastric corpus IL-8 concentration and neutrophil infiltration in duodenal ulcer patients. Aliment Pharmacol Ther 1997;11:793-800.

32 Daw MA, Deegan P, Leen E, et al. Short report: the effect of omeprazole on Helicobacter pylori and associated gastritis. Aliment Pharmacol Ther 1991;5:435-9.

33 Logan RP, Walker MM, Misiewicz JJ, et al. Changes in the intragastric distribution of Helicobacter pylori during treatment with omeprazole. Gut 1995;36:12-16.

34 McCloy RF, Arnold R, Bardhan KD, et al. Pathophysiological effects of long-term acid suppression in man. Dig Dis $S c i$ 1995;40:96S-120S.

35 Stolte M, Bethke B, Ruhl G, et al. Omeprazole-induced pseudohypertrophy of gastric parietal cells. $Z$ Gastroenterol 1992;30:134-8.

36 Driman DK, Wright C, Tougas G, et al. Omeprazole produces parietal cell hypertrophy and hyperplasia in humans. Dig Dis Sci 1996;41:2039-47.

37 Krishnamurthy S, Dayal Y. Parietal cell protrusions in gastric ulcer disease. Hum Pathol 1997;28:1126-30.

38 Genta RM, Graham DY. Depth of Helicobacter pylori in the oxyntic mucosa in patients treated with proton-pump inhibitors [abstract]. Gastroenterology 1998;114:A131.

39 Eissele R, Brunner G, Simon B, et al. Gastric mucosa during treatment with lansoprazole: Helicobacter pylori is a risk factor for argyrophil cell hyperplasia. Gastroenterology 1997;112:707-17.

40 Lamberts R, Creutzfeldt W, Struber HG, et al. Long-term omeprazole therapy in peptic ulcer disease: gastrin, endocrine cell growth, and gastritis. Gastroenterology 1993; 104:1356-70. 